\title{
11ß-Hydroxylase loss disrupts steroidogenesis and reproductive function in zebrafish
}

\author{
James A Oakes ${ }^{1,2}$, Lise Barnard³, Karl-Heinz Storbeck ${ }^{3}$, Vincent T Cunliffe ${ }^{2}$ and Nils P Krone ${ }^{1,2,4}$ \\ 1Department of Oncology \& Metabolism, School of Medicine, University of Sheffield, Sheffield, UK \\ ${ }^{2}$ The Bateson Centre, Department of Biomedical Science, Firth Court, University of Sheffield, Western Bank, Sheffield, UK \\ ${ }^{3}$ Department of Biochemistry, Stellenbosch University, Stellenbosch, South Africa \\ ${ }^{4}$ Department of Medicine III, University Hospital Carl Gustav Carus, Technische Universität Dresden, Dresden, Germany
}

Correspondence should be addressed to N P Krone: N.Krone@sheffield.ac.uk

\begin{abstract}
The roles of androgens in male reproductive development and function in zebrafish are poorly understood. To investigate this topic, we employed CRISPR/Cas9 to generate cyp11c1 (11ß-hydroxylase) mutant zebrafish lines. Our study confirms recently published findings from a different cyp11c1--- mutant zebrafish line, and also reports novel aspects of the phenotype caused by loss of Cyp11c1 function. We report that Cyp11c1deficient zebrafish display predominantly female secondary sex characteristics, but may possess either ovaries or testes. Moreover, we observed that cyp11c1-/- mutant male zebrafish are profoundly androgen- and cortisol-deficient. These results provide further evidence that androgens are dispensable for testis formation in zebrafish, as has been demonstrated previously in androgen-deficient and androgen-resistant zebrafish. Herein, we show that the testes of cyp11c1-/- mutant zebrafish exhibit a disorganised tubular structure; and for the first time demonstrate that the spermatic ducts, which connect the testes to the urogenital orifice, are severely hypoplastic in androgendeficient zebrafish. Furthermore, we show that spermatogenesis and characteristic breeding behaviours are impaired in cyp11 $\mathrm{c1}^{-/-}$mutant zebrafish. Expression of nanos2, a type A spermatogonia marker, was significantly increased in the testes of Cyp11c1deficient zebrafish, whereas expression of markers for later stages of spermatogenesis was significantly decreased. These observations indicate that in zebrafish, production of type A spermatogonia is androgen-independent, but differentiation of type A spermatogonia is an androgen-dependent process. Overall, our results demonstrate that whilst androgens are not required for testis formation, they play important roles in determining secondary sexual characteristics, proper organisation of seminiferous tubules, and differentiation of male germ cells.
\end{abstract}
Key Words
- zebrafish
- testes
- sperm
- androgen
- cyp11c1

Journal of Endocrinology (2020) 247, 197-212

\section{Introduction}

The roles of androgens in zebrafish sex differentiation, development of male sexual characteristics, and maintenance and function of the adult testes are poorly understood. Laboratory strains of zebrafish lack sex chromosomes and exhibit polygenic sex determination
(Liew et al. 2012). Prior to gonadal differentiation zebrafish develop a juvenile ovary; this is maintained in presumptive females and continues to develop, whereas presumptive males undergo juvenile ovary-to-testis transformation (Uchida et al. 2002, Wang et al. 2007). 
This process is highly sensitive to sex steroids. Treatment of developing fish with oestrogens results in feminisation (Andersen et al. 2003, Brion et al. 2004, Orn et al. 2016); whilst mutation of cyp19a1a, crucial for oestrogen production, causes robust masculinisation (Lau et al. 2016, Yin et al. 2017). Conversely, treatment of developing zebrafish with androgens results in robust masculinisation (Larsen \& Baatrup 2010, Morthorst et al. 2010, Lee et al. 2017). These findings suggest that androgen-deficiency or androgen-resistance might cause robust feminisation; however, this is not the case.

Recent studies have characterised androgen-deficient and -resistant zebrafish (Crowder et al. 2017, Yong et al. 2017, Tang et al. 2018, Li et al. 2020, Oakes et al. 2019). These fish share similar phenotypes, exhibiting primarily female secondary sex characteristics. Despite their appearance, these fish may possess either ovaries or testes, indicating that androgens are dispensable for testis differentiation. Androgen-deficient or -resistant male zebrafish are infertile in standard breeding scenarios, however, their sperm may fertilise eggs collected from WT females in IVF experiments. Several factors appear to contribute to this phenotype, including disorganised testicular structure and impaired breeding behaviour and spermatogenesis.

Steroid 11 $\beta$-hydroxylase (CYP11B1) is crucial for the conversion of 11-deoxycortisol to cortisol in the final stage of glucocorticoid biosynthesis in humans (Miller \& Auchus 2011). CYP11B subfamily enzymes are located at the inner-mitochondrial membrane, where they are supplied with electrons by NADPH via ferredoxin and ferredoxin reductase, to allow substrate hydroxylation (Schiffer et al. 2015). In zebrafish, the final stage of glucocorticoid biosynthesis is catalysed by the zebrafish homolog of 11ß-hydroxylase, Cyp11c1 (Fig. 1)
(Tokarz et al. 2015). Genomic analysis of CYP11 genes suggests that the $C Y P 11 C$ genes in fish and the $C Y P 11 B$ genes in terrestrial mammals are orthologous, which is consistent with studies on the evolution of adrenal and sex steroidogenic enzymes (reviewed in Baker et al. (2015)). Unlike the situation in mammals, Cyp11c1 is thought to play an important role in gonadal androgen synthesis in zebrafish (Fig. 1) (Oakes et al. 2019). In the zebrafish testes Cyp11c1 is found in the steroidogenic Leydig cells, as well as in certain germ cell stages (Caulier et al. 2015). The principal androgens in humans are testosterone and $5 \alpha$-dihydrotestosterone, whereas in zebrafish the principal androgen is 11-ketotestosterone (Tokarz et al. 2015). This is due to the fact that zebrafish favour production of 11-oxygenated androgens from androstenedione, rather than conversion of androstenedione to testosterone (de Waal et al. 2008).

Cyp11c1 activity depends on electron transfer from the steroidogenic cofactor Fdx1b (Griffin et al. 2016). Our recent work has established Fdx1b-deficient zebrafish as a model of combined androgen- and cortisol-deficiency. Fdx1b-deficient zebrafish are infertile and exhibit disorganised testis structure and impaired spermatogenesis, as well as reduced stereotypical breeding behaviours (Oakes et al. 2019).

Almost simultaneously with the submission of this manuscript, another paper was published describing a different zebrafish cyp11c1-mutant line (Zhang et al. 2020). This study focussed primarily on the phenotypic characteristics of cyp11c1-mutant zebrafish during development. Herein, we present cyp11c1-mutant zebrafish lines as novel models for research into the roles of steroid deficiency on sex differentiation and adult reproductive processes. Our study confirms key findings of the previously published cyp11c1-mutant line

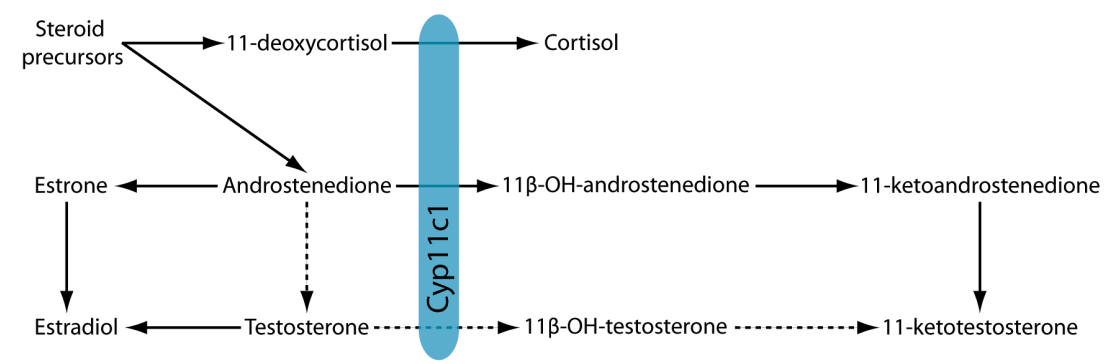

\section{Figure 1}

The roles of Cyp11c1 (11ß-hydroxylase) in androgen and glucocorticoid biosynthesis in zebrafish. Cyp11c1 is required for the final stage of glucocorticoid biosynthesis: the conversion of 11-deoxycortisol to cortisol. 11-Ketotestosterone, the principal zebrafish androgen, may be produced via two pathways beginning with androstenedione. The major pathway (solid arrows) involves 11ß-hydroxylation of androstenedione by Cyp11c1 to produce $11 \beta$-hydroxyandrostenedione, whereas the minor pathway (dashed arrows) requires 11 $\beta$-hydroxylation of testosterone by Cyp11c1 to produce $11 \beta$-hydroxytestosterone. A full colour version of this figure is available at https://doi.org/10.1530/JOE-20-0160. 
and also presents several novel, previously undescribed phenotypes. Our results confirm that Cyp11c1-deficient zebrafish are cortisol and androgen deficient, exhibit infertility and impaired breeding behaviour, as well as reduced spermatogenesis. Novel findings in our study include characterisation of steroid precursor concentrations, disorganised testis morphology in adult mutants and impaired locomotor behaviour. In addition to this, we have performed in-depth molecular investigation into the nature of impaired spermatogenesis in cyp11c1-/- mutant zebrafish. We also demonstrate, for the first time, that androgens are crucial for development or maintenance of key anatomical reproductive structures such as the spermatic duct, the structure linking the testes and urogenital orifice.

\section{Materials and methods}

\section{Zebrafish husbandry and ethics}

Adult zebrafish were maintained in a recirculating system (ZebTECTM, Tecniplast ${ }^{\circledR}$, Kettering, UK) at $28.5^{\circ} \mathrm{C}$ on a $10 \mathrm{~h}$ light:14 h darkness photoperiod. Zebrafish were bred from an AB WT background. Fish were aged between 96 and 154 days post fertilisation (dpf) at the time of experimentation. Fish were euthanized by administration of the anaesthetic tricaine mesylate (Pharmaq, Fordingbridge, UK). All experiments with animals were performed under licence from the UK Home Office and approved by the University of Sheffield Animal Welfare and Ethical Review Body (AWERB).

\section{Targeted genetic disruption of cyp11c1 by CRISPR/Cas9}

Mutation of cyp11c1 was achieved using the SygRNA system (Sigma). A crRNA was designed to target exon 2 of cyp11c1 (ENSDART00000185978.1). Approximately $1 \mathrm{~nL}$ of a $4 \mu \mathrm{L}$ mixture containing $0.1 \mu \mathrm{M}$ crRNA, $0.1 \mu \mathrm{M}$ tracrRNA (Sigma), $1 \mu \mathrm{L}$ phenol red and $1 \mu \mathrm{L}$ Cas9 (NEB, Ipswich, Massachusetts, USA) was injected into 1-cell stage embryos. The Cas9 cut site overlapped a BslI restriction site, allowing screening for mutant alleles lacking sensitivity to BslI (Supplementary Fig. 1, see section on supplementary materials given at the end of this article). CRISPR/Cas9-injected embryos were raised and outcrossed to unrelated WT fish. The resulting progeny were screened for disruption of cyp11c1, and out-of-frame mutations were identified by DNA sequencing.

\section{Steroid quantification by LC-MS/MS}

Adult zebrafish were euthanized, transferred to a silanised test tube, and snap-frozen on dry ice. Sample preparation was as previously described except for omission of the final Phree column elution (Oakes et al. 2019). Steroids were separated and quantified using an Acquity UPLC System (Waters, Milford, CT, USA) coupled to a Xevo TQ-S tandem mass spectrometer (Waters) as previously described (O'Reilly et al. 2017).

\section{Fertility and behavioural analysis, IVF and sperm release}

cyp11c1-/- mutant male zebrafish were outcrossed with unrelated WT females on three separate occasions using the pair mating technique, breeding was deemed successful if fertilised eggs were produced (Westerfield 2000). Breeding behaviour was analysed as previously described (Oakes et al. 2019). For open field tests fish were transferred individually to an opaque test tank and movement was tracked for 5 min using Zebralab software (Viewpoint, Lyon, France), this was repeated on three occasions with at least 3 days between trials. Fish were deemed to exhibit fast swimming behaviour at speeds of greater than $10 \mathrm{~cm} / \mathrm{s}$.

For in vitro fertilisation (IVF) and sperm counting, testes were dissected and lightly homogenised in a $50 x$ mass:volume dilution of Hank's balanced salt solution (HBSS). Eggs were obtained by gentle abdominal palpation of anaesthetised WT female fish. In total, $50 \mu \mathrm{L}$ of sperm solution was added to a clutch of eggs, followed by $400 \mu \mathrm{L}$ of aquarium water; after $2 \mathrm{~min}$ a further $2 \mathrm{~mL}$ of aquarium water was added (Westerfield 2000). Fertilisation was confirmed under a dissecting microscope. For sperm counting, $10 \mu \mathrm{L}$ of sperm solution was transferred to each chamber of an improved Neubauer haemocytometer (Hawksley, Sussex, UK). A minimum of 200 sperm was counted in each chamber and the number of sperm/nL was multiplied by the dilution factor $(50 \times)$ to obtain sperm counts. Gonadosomatic index was calculated using the formula GSI= (gonad weight/total tissue weight) $\times 100$.

To assess sperm release, cyp11c1-l- mutant and WT sibling zebrafish were anaesthetised, and semen was collected by stroking the abdomen with blunt-ended forceps (Millipore), followed by aspiration of expelled fluid into a microcapillary tube and transfer to $25 \mu \mathrm{L}$ of ice-cold HBSS (Westerfield 2000). Presence of mature sperm was confirmed by visualisation under a $20 \times$ objective. 


\section{Histology}

Preparation of samples and H\&E staining was performed as previously described (Oakes et al. 2019).

\section{Gene expression analysis by quantitative PCR (qPCR)}

For larval gene expression analysis, the progeny of a cyp $111^{+/-}$incross were sorted by visual background adaption (VBA) at 4-5 dpf (Griffin et al. 2016). Larvae were housed in dark conditions for $1 \mathrm{~h}$, followed by a 20 min light exposure. Larvae were subsequently sorted into lightly (VBA+) and darkly (VBA-) pigmented groups. Sorted larvae were pooled into groups of 20 and snapfrozen on dry ice. For adult gene expression analysis, fish were euthanized, dissected, and organs collected by snap freezing on dry ice.

Total RNA was isolated using Trizol (Ambion). cDNA was prepared using SuperScript II (Thermo Fisher Scientific) with 20mer oligo(dT) primers (IDT, Coralville, IA, USA) and $1 \mu \mathrm{g}$ of RNA. GoTaq qPCR master mix (Promega) was utilised in reactions containing $1 \mu \mathrm{L}$ cDNA synthesis product and specific primers (Supplementary Table 1) at $1000 \mathrm{nM}$. Reactions were run on a 7900HT Fast Real-Time PCR System (Applied Biosystems). Data were analysed by the Livak method (Livak \& Schmittgen 2001) with ef1a as reference gene. Fold changes in gene expression are displayed relative to expression in WT male tissue.

\section{Statistical methods}

Statistical analysis was performed in Graphpad Prism (GraphPad Software). Data normality was assessed using inbuilt tests. Normally distributed biometric and qPCR data were analysed using unpaired $t$-tests, non-normal data were analysed by Mann-Whitney tests. Behavioural data were analysed using multiple t-tests (Holm-Sidak method). Scatter plot error bars represent the s.E.M. Statistical significances are reported using asterisks: ${ }^{*} P<0.05,{ }^{* *} P<0.01,{ }^{* * *} P<0.001,{ }^{* * \star *} P<0.0001$, ns, not significant.

\section{Results}

\section{Generation of cyp11c1 mutant alleles by CRISPR/Cas9}

Exon 2 of cyp11c1 was targeted using the SygRNA twopart system (see 'Methods' section). CRISPR-injected fish were outcrossed at $\sim 10 \mathrm{wpf}$, and heritable mutations were identified in their progeny. Several out-of-frame mutations were identified, including 11 bp (c.312_322del, p.Glu105Profs*33, allele number SH548) and 47bp (c.285_331del, p.Met96Hisfs*30, allele number SH547) deletions. Both are predicted to produce a truncated protein $\sim 25 \%$ the size of the WT isoform (Supplementary Fig. 2). Inheritance of cyp11c1 mutant alleles did not significantly deviate from expected Mendelian ratios.

Cortisol-deficient zebrafish larvae exhibit impaired VBA - the ability to adapt pigmentation to light conditions. We found that VBA was impaired in cyp11c1-1- mutant larvae at $5 \mathrm{dpf}$, and expression of glucocorticoid responsive genes fkbp5 and pck1 was significantly decreased, suggesting reduced cortisol production due to loss of Cyp11c1 function (Supplementary Fig. 3 and Supplementary Table 2).

\section{cyp11c1-/- mutant zebrafish exhibit female secondary sex characteristics but may possess either ovaries or testes}

Morphological secondary sex characteristics in zebrafish include body shape, fin and body pigmentation, and genital papilla prominence. Male zebrafish are streamlined in shape, have palely pigmented dorsal fins, and orange striped anal fins. Female fish have a more rounded abdomen, greenyellow pigmented dorsal fins and little orange pigmentation in the anal fin (Fig. 2). Female zebrafish have a large and prominent genital papilla; in males this structure is much smaller and mostly hidden from view.

Upon raising the progeny of $c y p 11 c 1^{+/-}$incrosses, it was apparent that all homozygous mutant fish displayed predominantly female secondary sex characteristics (Fig. 2). Close inspection revealed that some $c y p 11 c 1^{-/-}$mutant fish had prominent genital papillae like WT females, whereas others had small hidden genital papillae like those of WT males. Dissection of $c y p 11 c 1^{-/-}$mutant fish revealed that they could possess either testes or ovaries, and this was accurately predicted by the presence or absence of a prominent genital papilla. The ratio of males:females (testes:ovary) in populations of WT and cyp11c1-/- zebrafish did not significantly differ.

Additionally, biometric data was also collected. Males from both cyp11c1-/- mutant lines were significantly longer and heavier than WT siblings (Fig. 2).

\section{Adult cyp11c1-/- mutant male zebrafish exhibit profound cortisol and 11-ketotestosterone deficiency}

In order toassess theimpact of cyp11c1 mutation oninterrenal and testicular steroidogenesis, we employed LC-MS/MS to quantify steroid concentrations in samples prepared from 


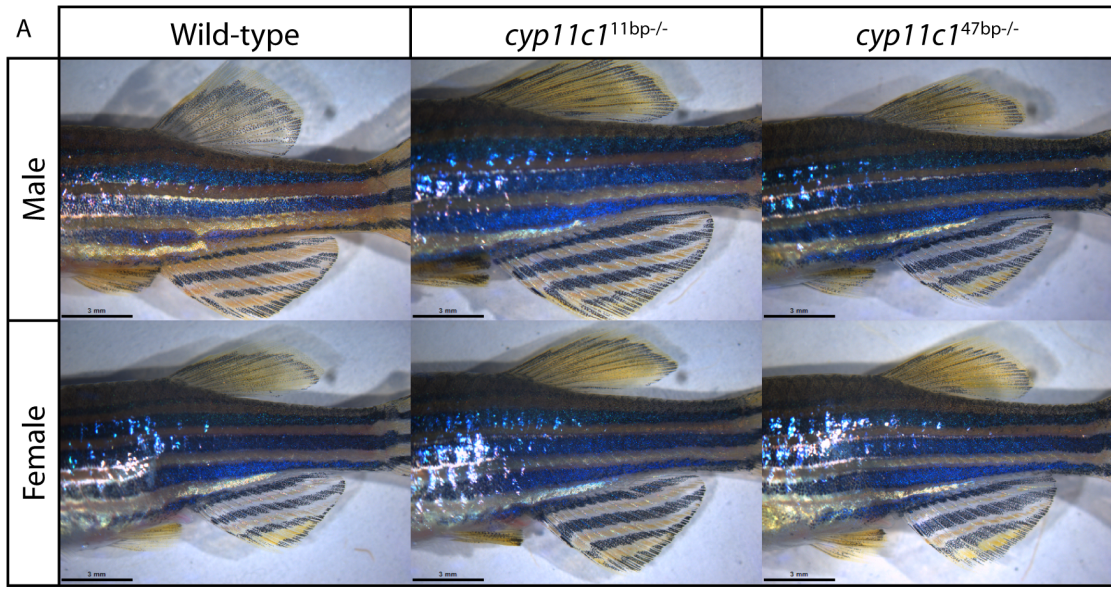

B Length-cyp11c111bp C Length-cyp11c147bp D Weight-cyp11c111bp E Weight-cyp11c147bp
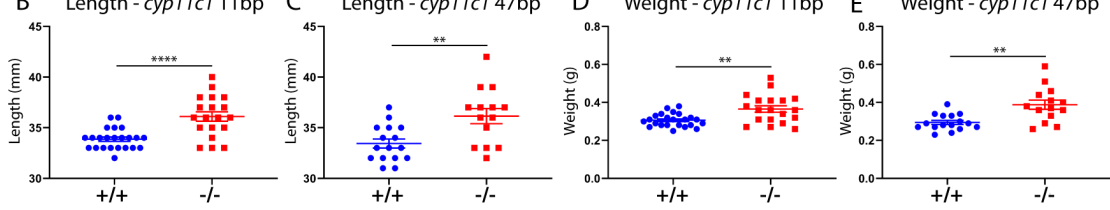

\section{Figure 2}

Feminisation of secondary sex characteristics and increased length and weight in cyp11c1--- mutant male zebrafish. (A) cyp11c1--- mutant male zebrafish exhibited fin pigmentation patterns more commonly seen in female zebrafish. WT male zebrafish exhibited pale dorsal fins and strongly orange striped anal fins. cyp11 $\mathrm{c}^{-1-}$ mutant male zebrafish exhibited reduced orange pigmentation in the anal fin and green-yellow pigmentation in the dorsal fin, like that seen in WT females. (B, C, D and E) Length and weight were significantly increased in homozygous mutants from both cyp11c1 mutant alleles (B and D) $11 \mathrm{bp}:$ WT $n=23$ mutant $n=19$, length $P \leq 0.0001$ weight $P=0.0016$. ( $C$ and E) $47 \mathrm{bp}$ : WT $n=16$ mutant $n=14$, length $P=0.0034$ weight $P=0.0010$ ). Statistical analysis was by unpaired

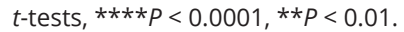

whole adult zebrafish males. Cortisol concentrations were profoundly decreased by mutation of cyp11c1 (Fig. 3), whereas concentrations of its precursor, 11-deoxycortisol, were significantly increased. Thus, we have demonstrated the in vivo importance of Cyp11c1 function for the conversion of 11-deoxycortisol to cortisol in glucocorticoid biosynthesis. Concentrations of the sex steroid precursor androstenedione were significantly increased in $c y p 11 \mathrm{c1}^{-/-}$
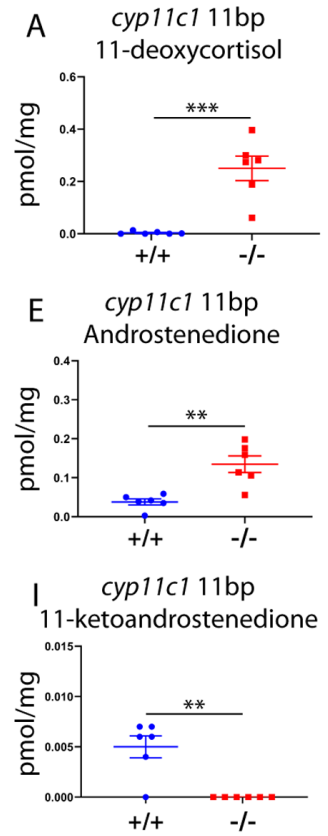
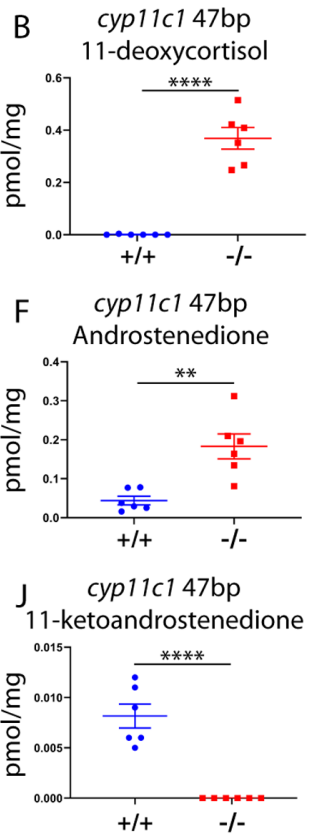
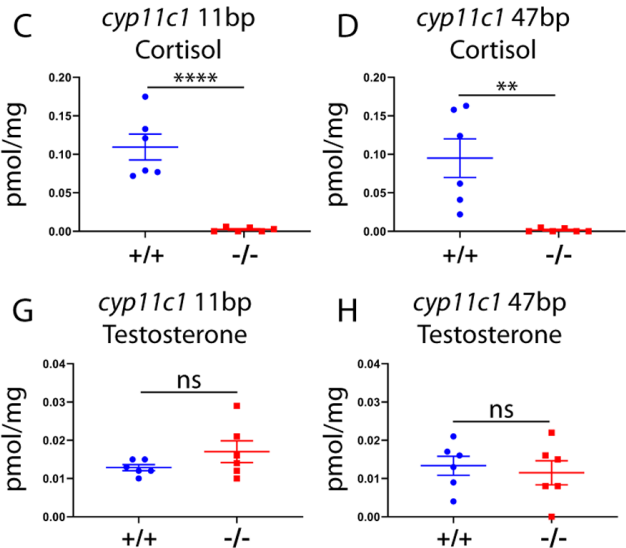

H cyp11c1 47bp

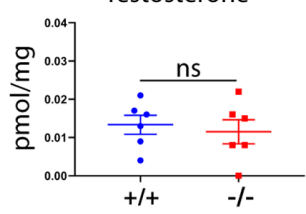

L cyp11c1 47bp

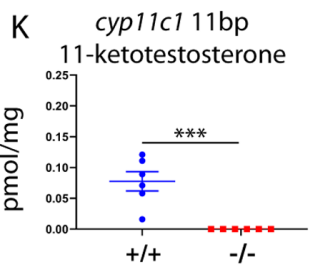

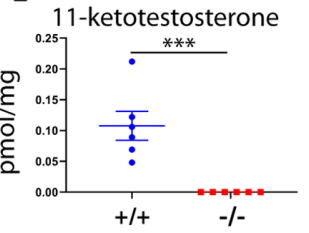

Figure 3

The steroid profile of adult male cyp11c1-/- mutant zebrafish is consistent with 11 $\beta$-hydroxylase deficiency. Steroid hormone concentrations were quantified in whole adult zebrafish $(n=6)$ by LC-MS/MS. Concentrations of cortisol (C and D) $(11$ bp: $P<0.0001,47$ bp: $P=0.0039)$ were profoundly reduced in cyp11c1-/- mutant zebrafish, whereas concentrations of the cortisol precursor $11-$ deoxycortisol $(A$ and $B)(11 \mathrm{bp}: P=0.0003,47 \mathrm{bp}: P<0.0001)$ were significantly increased. Concentrations of the sex steroid precursor androstenedione $(\mathrm{E}$ and $\mathrm{F})(11 \mathrm{bp}: P=0.0017,47 \mathrm{bp}: P=0.0021)$ were significantly increased in cyp11c1-/- mutant zebrafish, whereas concentrations of 11-ketotestosterone (K and L) $(11 \mathrm{bp}: P=0.0006,47 \mathrm{bp}: P=0.0010)$ and the intermediate steroid, 11-ketoandrostenedione ( $\mathrm{and}$ ) (11 bp: $P=0.0010,47 \mathrm{bp}: P<0.0001$ ), were undetectable in mutant zebrafish. Concentrations of testosterone ( $\mathrm{G}$ and $\mathrm{H})(11 \mathrm{bp}: P=0.1895,47 \mathrm{bp}: P=0.6581)$ were not affected by mutation of $c y p 11 \mathrm{c} 1$. Results analysed by unpaired $t$-tests, $* \star P<0.01$, $\star \star \star P<0.001, * \star * \star P<0.0001$. A full colour version of this figure is available at https://doi.org/10.1530/JOE-20-0160. 
mutant male zebrafish, probably due to blocking of the androgen synthesis pathway; shunting of glucocorticoid precursors into the sex steroid pathway may also contribute to increased androstenedione concentrations. Blockade of the androgen synthesis pathway was evidenced by undetectable concentrations of 11-ketotestosterone and its precursor 11-ketoandrostenedione (Fig. 3). Concentrations of testosterone were not affected by mutation of cyp11c1 (Fig. 3).

Expression of the glucocorticoid-responsive genes fkbp5 and pck1 (Griffin et al. 2016, Eachus et al. 2017) was significantly reduced in cyp11c1-/- mutant male zebrafish livers compared to WT siblings, thus demonstrating the
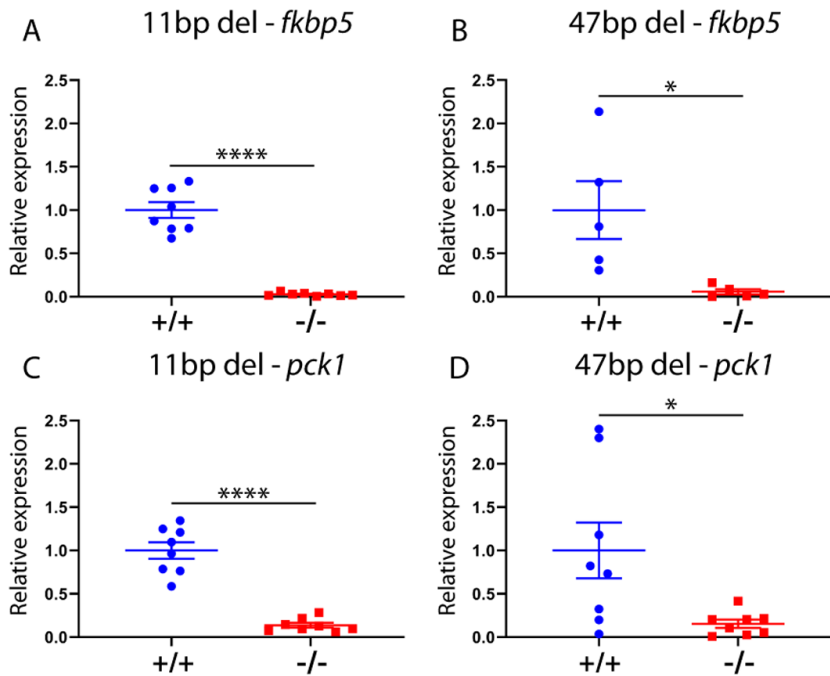

D

47bp del - pck1

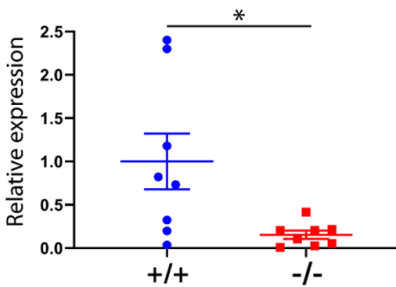

E $\quad 11 b p$ del-cyp2k22
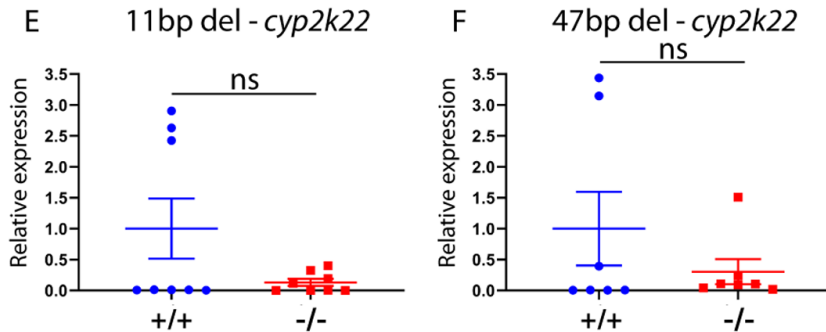

\section{Figure 4}

Expression of steroid responsive genes was decreased in the livers of Cyp11c1-deficient male zebrafish. Expression of steroid responsive genes in cyp11c1-/- mutant male zebrafish and WT siblings was quantified by qPCR with ef1 $a$ as the reference gene. Expression of robustly glucocorticoid responsive genes fkbp5 (11 bp WT $n=8$, mutant $n=8$, $P<0.0001 ; 47$ bp WT $n=5$, mutant $n=5, P=0.0230$ ) and $p c k 1$ (11 bp WT $n=8$, mutant $n=8, P<0.0001,47$ bp WT $n=8$, mutant $n=8, P=0.0212$ ) was significantly reduced in the livers of cyp11c1-/- mutant male zebrafish. Quantification of the expression of cyp2k22 (11 bp WT $n=8$, mutant $n=8$, $P=0.2345,47 \mathrm{bp} \mathrm{WT} n=7$, mutant $n=7, P=0.6200$, Mann-Whitney tests), a proposed androgen responsive gene, revealed an apparent reduction in expression in cyp11c1-/- mutant male zebrafish, however, this did not achieve statistical significance. Data analysed by unpaired $t$-tests unless otherwise stated, $* P<0.05, * \star \star \star P<0.0001$. A full colour version of this figure is available at https://doi.org/10.1530/JOE-20-0160.

(c) 2020 Society for Endocrinology Published by Bioscientifica Ltd. Printed in Great Britain systemic consequences of glucocorticoid deficiency. An apparent decrease in the expression of the proposed androgen-responsive gene $c y p 2 k 22$, which is postulated to play a role in androgen metabolism, (Fetter et al. 2015, Siegenthaler et al. 2017) did not achieve statistical significance (Fig. 4).

\section{Disruption of cyp11c1 results in infertility and impaired breeding behaviour}

Androgen-resistant and androgen-deficient male zebrafish are infertile (Crowder et al. 2018, Oakes et al. 2019) and the incidence of stereotypical breeding behaviours is decreased (Yong et al. 2017). To investigate the impact of Cyp11c1-deficiency on breeding behaviour in male zebrafish, we analysed two well-characterised breeding behaviours. In all trials, the number of intimate contacts, where fish touch or cross one another, and the duration of chasing, where one fish closely follows the other, were significantly reduced in cyp11c1-l- mutant lines compared to WT siblings (Fig. 5). The proportion of trials resulting in the production of fertilised embryos was also recorded. Outcrosses of WT females and WT sibling males from the 11 and $47 \mathrm{bp}$ alleles produced fertilised embryos in $92 \%$ and $66 \%$ of crosses, respectively. No fertilised embryos were observed in any crosses with cyp11c1-/mutant zebrafish (Table 1). Despite exhibiting infertility in normal breeding scenarios, the sperm of cyp11c1-/mutant zebrafish were able to fertilise eggs collected from WT females by IVF (Table 2).

Whilst conducting breeding experiments on cyp11c1--- mutant zebrafish it was noted that they appeared to exhibit reduced locomotor activity. In order to quantify this, mutant and WT male zebrafish were exposed to open field tests. This revealed that the total distance swam, as well as the duration of fast swimming, was significantly and consistently reduced in cyp11c1-/mutant males (Fig. 6). Freezing duration, the duration for which the fish was stationary in the tank, was also recorded. Freezing duration was consistently greater in trials involving cyp11c1-/- mutant zebrafish; however, these results were not statistically significant, presumably due to the extremely high variability with which fish express this phenotype (Fig. 6).

\section{Cyp11c1 disruption results in testicular disorganisation and reduced spermatogenesis}

In order to examine the impact of cyp11c1 mutations on testis morphology, we collected coronal sections through 
A
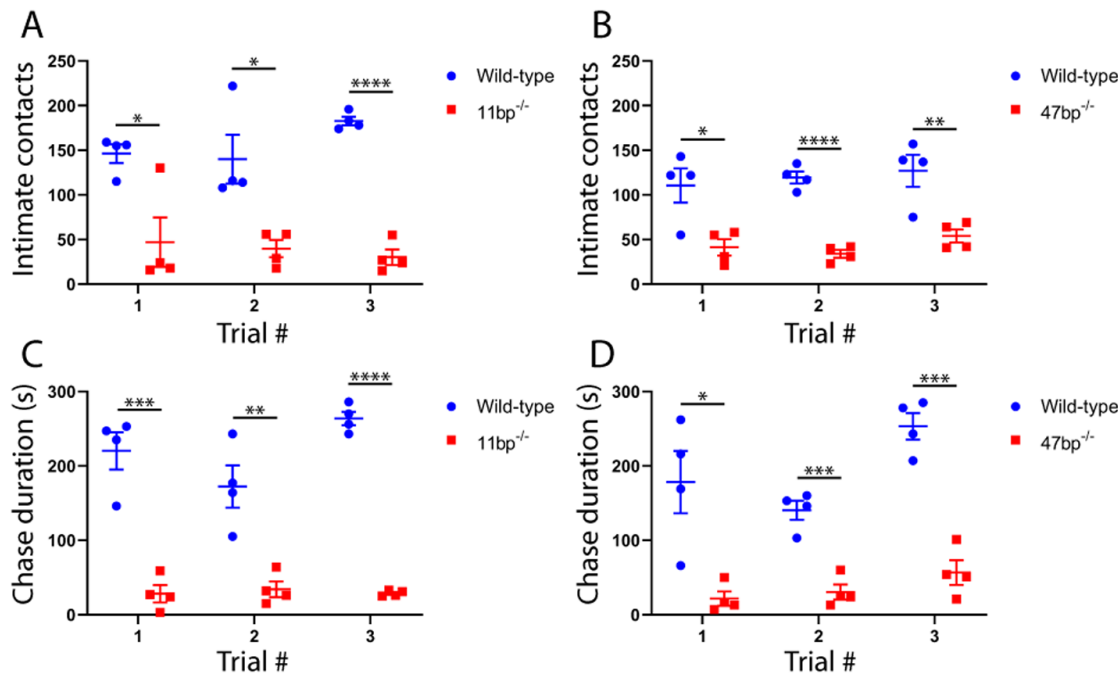

$D$

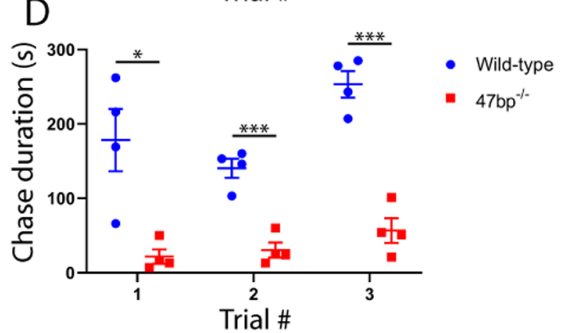

\section{Figure 5}

Stereotypical breeding behaviours are reduced in pairings with Cyp11c1-deficient male zebrafish. cyp11c1-- mutant $(n=4)$ and WT sibling male $(n=4)$ zebrafish were paired with WT females and behaviour was analysed during the first 5 min of breeding. This procedure was repeated on three separate occasions. Intimate contacts ( $\mathrm{A}$ and $\mathrm{B}$ ) (11 bp: $1, P=0.0270 ; 2, P=0.0270 ; 3, P<0.0001$; 47 bp: 1, $P=0.0184 ; 2, P=0.0001,3, P=0.0184)$ and chasing duration ( $C$ and $D)(11 \mathrm{bp}: 1$, $P=0.0009 ; 2, P=0.0038 ; 3, P<0.0001 ; 47 \mathrm{bp}: 1$ $P=0.0109 ; 2, P=0.0011 ; 3, P=0.0006$ ) were significantly reduced in all trials and in both 11 and $47 \mathrm{bp}$ deletion alleles. Results analysed using multiple $t$-tests with the Holm-Sidak method, $\star P<0.05, * * P<0.01, * \star * P<0.001, * \star \star * P<0.0001$ A full colour version of this figure is available at https://doi.org/10.1530/JOE-20-0160. whole adult zebrafish and performed H\&E staining. The testes of WT males appeared to be well organised, with defined seminiferous tubule structures clearly visible (Fig. $7)$. In contrast, the structure of $c y p 11 c 1^{-/-}$mutant testes was generally disorganised, with defined seminiferous tubule structures rarely in evidence. The seminiferous tubules of WT testes comprised clusters of developing spermatogonia, spermatocytes and spermatids lining the perimeter, with mature spermatozoa in the central lumen (Fig. 7). The testes of $c y p 11 c 1^{-1-}$ mutant zebrafish also contained cells at all stages of spermatogenesis; however, the proportion of developing germ cells to mature sperm appeared to be much greater. This was accompanied by a qualitative reduction in the amount of mature spermatozoa; this finding was later quantified by sperm counting (Fig. 7). No difference in gonadosomatic index, the percentage contribution of the gonads to body weight, was recorded for either $c y p 11 c 1^{-/-}$mutant allele.

\section{Cyp11c1 is crucial for development of the spermatic duct}

In zebrafish, sperm is conducted from the testes to the urogenital orifice via the spermatic duct (Menke et al. 2011). As testicular tubule structure was found to be disorganised in cyp11c1-/- mutant zebrafish, we investigated the possibility that the spermatic duct may also exhibit impaired development or maintenance.

The structure of the spermatic duct was examined ventral to the spermatogenic tissue of the testes and dorsal to the genital orifice and was found to occupy the region posterior to the intestine and anterior to the renal collecting duct (Fig. 8). The spermatic ducts of WT zebrafish comprised an extensive tubular structure, with tubules containing spermatozoa (Fig. 8A and D). In contrast, the spermatic ducts of $c y p 11 c 1^{-/-}$mutant zebrafish appeared as severely hypoplastic structures immediately posterior to the intestine. cyp11c1-/- mutant spermatic ducts either contained no sperm (11 bp deletion: 4/5, 47 bp deletion: 2/5) (Fig. 8B and E) or existed as a slightly more extensive structure containing some mature spermatozoa (Fig. 8C and $\mathrm{F}$ ).

In order to determine if hypoplasia of the spermatic duct resulted in impaired sperm release, we subjected cyp11c1-/- mutant zebrafish and WT siblings to manual gamete expression (Westerfield 2000). Cyp11c1-deficient zebrafish exhibited profoundly impaired sperm release, although spermatozoa were observed in samples obtained from some cyp11c147bp-/- mutant zebrafish in one of two trials (Table 3 ).

Table 1 Infertility in cyp11c1-/- mutant male zebrafish.

\begin{tabular}{|c|c|c|c|}
\hline Allele & Genotype & Total number of crosses & Number of crosses resulting in fertilised eggs \\
\hline \multirow[t]{2}{*}{ 11bp deletion } & $+/+(n=4)$ & 12 & $11(92 \%)$ \\
\hline & $-1-(n=4)$ & 12 & $0(0 \%)$ \\
\hline \multirow[t]{2}{*}{ 47bp deletion } & $+/+(n=4)$ & 12 & $8(66 \%)$ \\
\hline & $-1-(n=4)$ & 12 & $0(0 \%)$ \\
\hline
\end{tabular}

cyp11c1-/- mutant $(n=4)$ and WT sibling males $(n=4)$ were outcrossed with WT females on three separate occasions. No crosses involving cyp11c1-/mutant males from either the 11 bp or 47 bp deletion alleles produced any fertilised embryos; their WT siblings produced fertilised embryos in $92 \%$ and $66 \%$ of crosses, respectively. 
Table 2 Proportion of cyp11c1-/- mutant and WT sibling sperm samples producing fertilised embryos in IVF experiments.

\begin{tabular}{llc}
\hline Genotype & & Proportion producing fertilised embryos \\
\cline { 1 - 1 } cyp11c111bp+/+ $(n=4)$ & $4 / 4(100 \%)$ \\
cyp11c111bp-/- $(n=4)$ & $3 / 4(75 \%)$ \\
cyp11c147bp+/+ $(n=4)$ & $4 / 4(100 \%)$ \\
cyp11c147bp-/- $(n=4)$ & $4 / 4(100 \%)$ \\
\hline
\end{tabular}

Testes were dissected from male zebrafish and homogenised in a 50x mass:volume dilution of HBSS. Sperm solutions were used to fertilise eggs collected from WT females.

\section{Reduced expression of pro-male and spermatogenic genes in the testes of cyp11c1-/- zebrafish}

Steroid hormones act via their cognate nuclear receptors to regulate gene transcription (de Waal et al. 2008). In order to understand the impact of altered steroid concentrations on gene expression in the testes, and gain insight into the molecular mechanisms underlying the observed phenotype, we used qPCR to measure the expression of genes related to gonadal function.

Igf3 and Insl3 are important factors in zebrafish spermatogenesis; specifically, they are involved in regulating the proliferation and differentiation of type A spermatogonia (Nobrega et al. 2015, Assis et al. 2016, Morais et al. 2017). Both igf3 and insl3 were significantly downregulated in cyp11c1-/mutant zebrafish, potentially suggesting impairment of early stages of spermatogenesis in these mutants (Fig. 9).
The expression of dmrt1 and sox $9 a$, both of which play important roles in male sex differentiation (Sun et al. 2013, Webster et al. 2017) was unaffected by Cyp11c1deficiency (Fig. 9). Expression of the androgen receptor, via which 11-ketotestosterone exerts its effects on gene expression, was significantly upregulated in the testes of cyp11c1-/- mutant zebrafish (Fig. 9). This indicates a potential compensatory mechanism involving increased androgen receptor expression to scavenge for reduced androgens. Inhibins exert negative feedback on the hypothalamus-pituitary-gonadal axis, and may also play a role in Sertoli cell proliferation and spermatogenesis in vertebrates (Gregory \& Kaiser 2004, Poon et al. 2009, Cai et al. 2011). We observed significant down-regulation of inha in Cyp11c1-deficient zebrafish (Fig. 9).

\section{Expression of spermatogenesis marker genes suggests a crucial role for androgens in the differentiation of type A spermatogonia into meiotic spermatocytes}

Spermatogenesis comprises a series of cell division and differentiation events whereupon spermatogonial stem cells give rise to primary spermatocytes, which then enter meiosis, and eventually give rise to mature haploid spermatozoa. Having observed a reduction in the numbers of mature spermatozoa by histology and sperm counting, we endeavoured to deepen our understanding of the impact of androgen deficiency
A Total distance swam

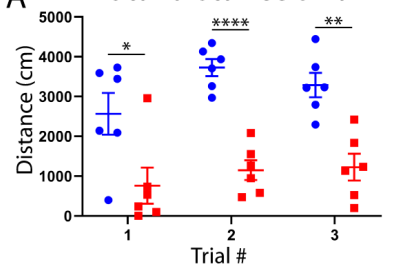

$C^{250}$ Fast swim duration

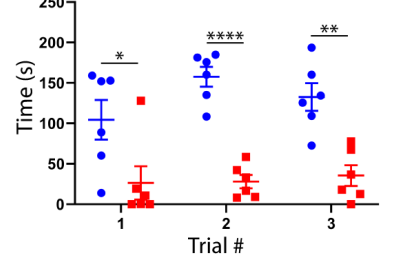

$E_{400} \quad$ Freeze duration

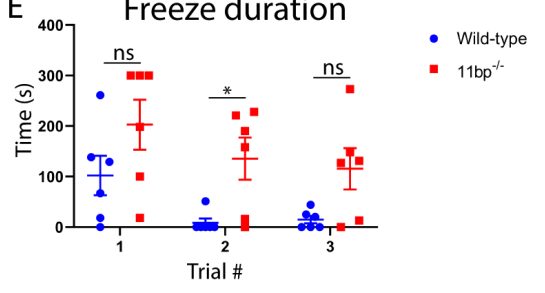

- Wild-type

- $11 \mathrm{bp}^{-\gamma-}$

- Wild-type
B Total distance swam

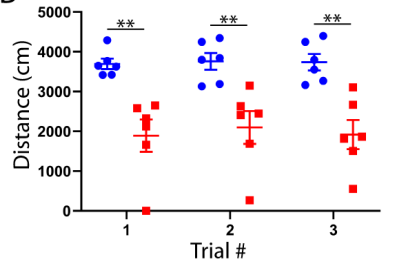

- Wild-type

- $47 \mathrm{bp}^{-1}$

$D_{250}$ Fast swim duration

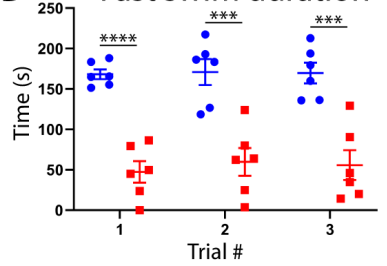

- Wild-type

- $47 \mathrm{bp}^{-/-}$

F

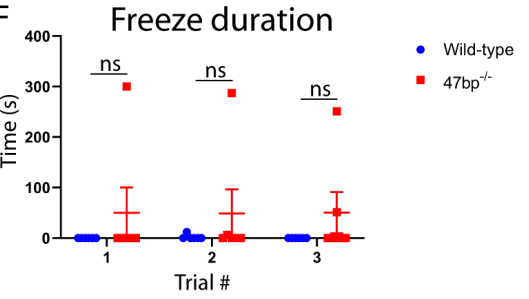

Figure 6

Cyp11c1-deficient male zebrafish exhibit decreased locomotor activity. In order to assess locomotion, cyp11c1-l- mutant male zebrafish and WT siblings $(n=6)$ were exposed to open field tests. Tests were repeated on three occasions, and total distance swam, fast swimming duration and freezing duration were recorded. Total distance swam (A and B) (11 bp: 1, $P=0.0265 ; 2$, $P<0.0001 ; 3, P=0.0021 ; 47$ bp: $1, P=0.0045,2$, $P=0.0050,3, P=0.0045)$ and fast swim duration (C and D) (11 bp: 1, $P=0.0348 ; 2, P<0.0001 ; 3$, $P=0.0021 ; 47$ bp: $1, P<0.0001 ; 2, P=0.0009 ; 3$, $P=0.0009$ ) were consistently reduced in both cyp11c1-1- mutant alleles across all trials. Freeze duration (E and F) (11 bp: 1, $P=0.1418 ; 2$, $P=0.0402 ; 3, P=0.0708 ; 47$ bp: $1, P=0.5740 ; 2$, $P=0.5740 ; 3, P=0.5740$ ) was consistently increased in cyp11c1-1- mutant male zebrafish, however, this was only significant in one trial $(E)$. Results were analysed using multiple $t$-tests with the Holm-Sidak method, $* P<0.05, * \star P<0.01$, $\star * \star P<0.001, * \star * \star P<0.0001$. A full colour version of this figure is available at https://doi. org/10.1530/JOE-20-0160. 

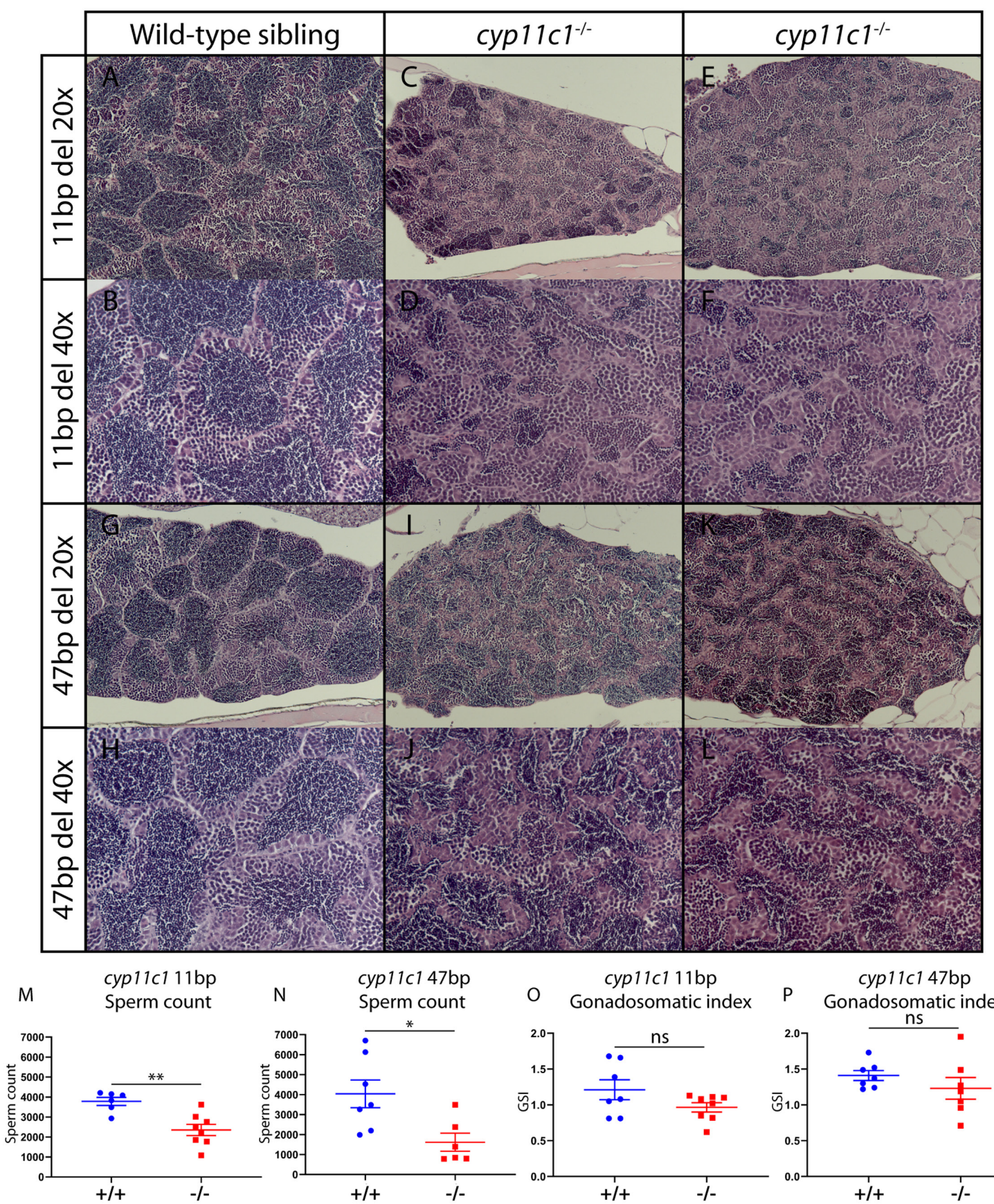

O cyp11c111bp

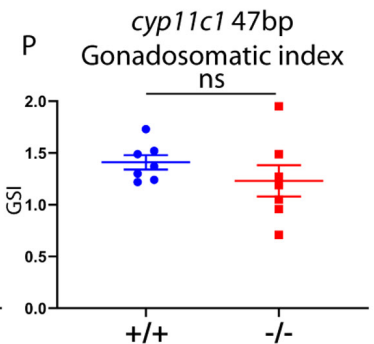

\section{Figure 7}

Mutation of cyp11c1 results in disorganised seminiferous tubules and reduced numbers of spermatozoa. The morphology and composition of cyp11c1-/ mutant ( $n=5$ per allele) and WT sibling ( $n=3$ per allele) testes was assessed by H\&E staining. The testes of WT sibling fish (A, B, G, H) contained defined seminiferous tubules whereas cyp11 c1-/- testes $(C, D, E, F ; I, J, K, L)$ contained poorly defined seminiferous tubules and comparatively fewer mature sperm. cyp11c1 mutant zebrafish exhibited significantly reduced sperm counts compared to WT siblings (M and N) (11 bp WT $n=6$, mutant $n=8$, $P=0.0023 ; 47 \mathrm{bp} \mathrm{WT} n=7$, mutant $n=6, P=0.0167$ ). No change in GSI was observed (O and P) (11 bp WT $n=7$, mutant $n=8, P=0.1201 ;$ WT $n=7,47 \mathrm{bp}$ mutant $n=7, P=0.3046$ ). Data analysed by unpaired $t$-tests, $* P<0.05, * * P<0.01$.

on spermatogenesis. To this end, we measured the expression of marker genes for several stages of spermatogenesis (Fig. 10). nanos 2 and piwil1 are expressed in type A spermatogonia (Beer \& Draper 2013, Chen et al.
2013, Safian et al. 2016). Significantly increased expression of nanos 2 was observed in cyp11c1-/- mutant zebrafish. Increased expression of piwil1 was observed in cyp11c1-/mutant zebrafish carrying the 11 bp deletion allele, 


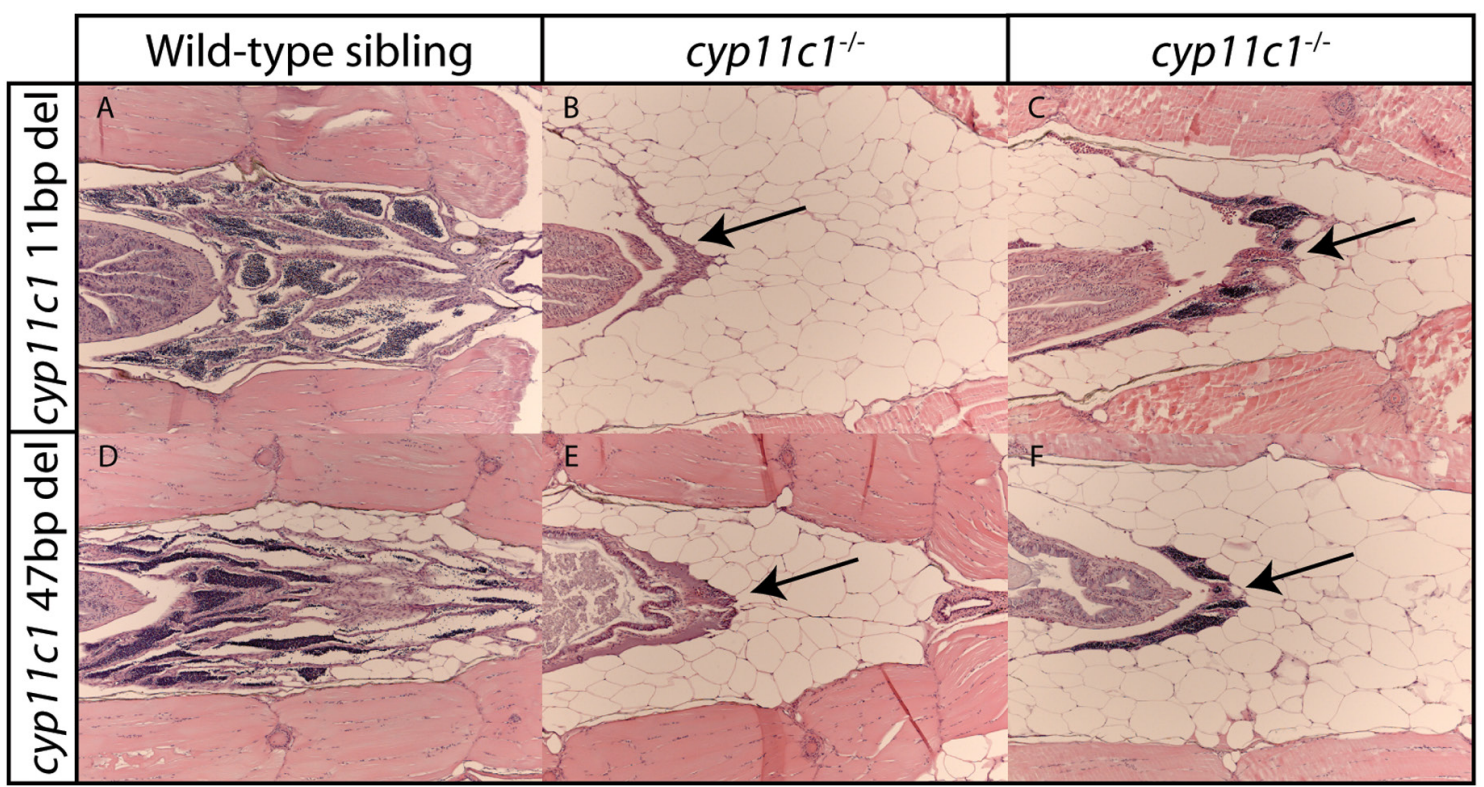

\section{Figure 8}

The spermatic ducts of cyp11c1-/- mutant zebrafish are hypoplastic. (A and D) Spermatic ducts of WT sibling zebrafish appeared as extensive networks of spermatozoa containing tubules situated between the intestine and renal collecting duct. (B, C, E, F) Spermatic ducts of cyp11c1-l- mutant zebrafish were comparatively smaller compared to those of WT siblings. cyp11 $11^{-/-}$spermatic ducts (arrows) appeared immediately posterior to the intestine and were severely underdeveloped, though some contained mature sperm. Images captured at 10× magnification.

but not in those carrying the 47bp deletion allele (Fig. 10). No change in the expression of the type B spermatogonia marker dazl (Chen et al. 2013) was observed; however, expression of the spermatocyte marker sycp3 (Ozaki et al. 2011) and spermatid marker odf3b (Yano et al. 2008, Nobrega et al. 2015) was significantly reduced in cyp11c1-/- mutant zebrafish, indicating a reduced proportion of cells at the later stages of spermatogenesis (Fig. 10). Taken together, these results indicate an accumulation of type A spermatogonia in cyp11c1-/mutant testes, due to the blockade or impairment of the transformation of spermatogonia into spermatocytes and subsequently spermatozoa.

\section{Discussion}

Herein, we described the phenotype of androgen- and cortisol-deficient cyp11c1 mutant zebrafish, paying particular attention to the roles of these steroids in the development, maintenance and function of the male reproductive system. We produced cyp11c1-/mutant alleles using CRISPR/Cas9 to target exon 2 of ENSDART00000185978.1 (Supplementary Fig. 2), whereas the previously published cyp11c1-mutant zebrafish line used CRISPR to target exon 3 of this transcript (Zhang et al. 2020). These mutations are predicted to produce similar truncated and functionally inactive protein products. (c) 2020 Society for Endocrinology Published by Bioscientifica Ltd. Printed in Great Britain
Cyp11c1 is important for the production of cortisol and 11-ketotestosterone (11KT), the principal zebrafish androgen (de Waal et al. 2008). Cyp11c1-deficient zebrafish exhibit profound deficiencies of both steroids, confirming the crucial role of Cyp11c1 in steroidogenesis (Fig. 3) (Zhang et al. 2020). In addition to measuring cortisol and $11 \mathrm{KT}$, we measured the concentrations of several intermediate steroid hormone precursors. We observed accumulation of 11-deoxycortisol and androstenedione (Fig. 3); precursors that may enter other steroidogenic pathways such as the oestrogen biosynthetic pathway. The phenotypic impact of the shunting of steroid precursors into alternative pathways remains unknown. Unchanged concentrations of testosterone in Cyp11c1deficient zebrafish suggest that excess androstenedione was not converted to testosterone; this provides in vivo

Table 3 Proportion of fish producing sperm samples during gamete expression experiments.

\begin{tabular}{|c|c|c|}
\hline \multirow[b]{2}{*}{ Genotype } & \multicolumn{2}{|c|}{ Number of fish producing sperm sample } \\
\hline & First trial & Second trial \\
\hline cyp11c111bp+/+ & $6 / 6$ & $8 / 8$ \\
\hline cyp11c111bp-/- & $0 / 6$ & $0 / 7$ \\
\hline cyp11c147bp+/+ & $6 / 6$ & $6 / 6$ \\
\hline сур11с147bp-/- & $6 / 9^{a}$ & $0 / 6$ \\
\hline
\end{tabular}

a2/6 samples from fish which produced sperm contained negligible sperm numbers. 

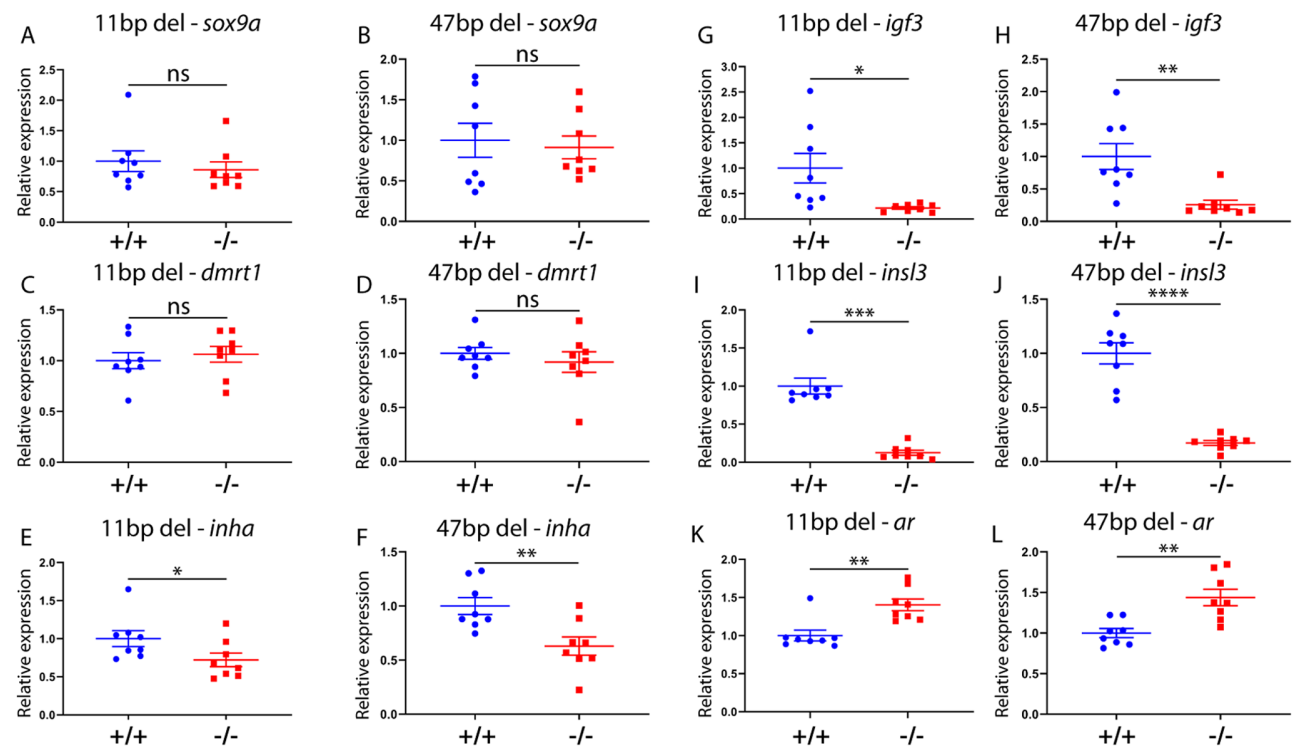

\section{Figure 9}

Expression of pro-male and spermatogenic genes in the testes of cyp11 c1-/- zebrafish. The expression of pro-male transcription factors sox9a (A and B) (11 bp $n=8, P=0.4418$, Mann-Whitney test; $47 \mathrm{bp} n=8, P=0.7337$ ) and dmrt1 ( $\mathrm{C}$ and $\mathrm{D}$ ) (11 bp $n=8, P=0.5802 ; 47 \mathrm{bp} n=8, P=0.4756)$ was unchanged in Cyp11c1-deficient zebrafish. Expression of the hypothalamus-pituitary-gonadal axis regulator inhibin alpha (inha) was significantly reduced in cyp11c1 mutant zebrafish ( $\mathrm{E}$ and $\mathrm{F}$ ) (11 bp $n=8, P=0.0499$, Mann-Whitney test; $47 \mathrm{bp} n=8, P=0.0062$ ). Expression of the spermatogenic factors igf3 (G and $\mathrm{H}$ ) (11 bp $n=8, P=0.0183 ; 47 \mathrm{bp} n=8, P=0.0011$, Mann-Whitney test) and insl3 (I and J) (11 bp $n=8, P=0.0002$, Mann-Whitney test; $47 \mathrm{bp} n=8$, $P<0.0001$ ) was profoundly reduced in the testes of cyp11 c1-/- mutant zebrafish. Expression of the androgen receptor (ar) was significantly upregulated in cyp11c1-I- mutant zebrafish testes ( $\mathrm{K}$ and L) (11 bp $n=8, P=0.0047$, Mann-Whitney test; $47 \mathrm{bp} n=8, P=0.0020$ ). Data analysed by unpaired $t$-tests unless otherwise specified, ${ }^{*} P<0.05,{ }^{*} * P<0.01,{ }^{*} * * P<0.001, * \star * * P<0.0001$. A full colour version of this figure is available at https://doi.org/10.1530/ JOE-20-0160.

evidence for previous in vitro findings indicating that conversion of androstenedione to testosterone, followed by $11 \beta$-hydroxylation of testosterone by Cyp11c1 to produce the $11 \mathrm{KT}$ precursor $11 \beta$-hydroxytestosterone, is a minor pathway to $11 \mathrm{KT}$ production in zebrafish (de Waal et al. 2008).

Decreased cortisol concentrations were reflected in decreased expression of the glucocorticoid responsive genes $f k b p 5$ and pck1 (Griffin et al. 2016, Eachus et al. 2017) in cyp11c1-/- mutant male liver tissue, demonstrating systemic glucocorticoid deficiency (Fig. 4). cyp2k22 has been proposed as an androgen-responsive gene in zebrafish (Fetter et al. 2015, Siegenthaler et al. 2017), and is robustly downregulated in the livers of androgendeficient $f d x 1 b^{-/-}$mutant zebrafish (Oakes et al. 2019). An apparent reduction in the expression of $c y p 2 k 22$ in the livers of $c y p 11 c 1^{-/-}$mutant zebrafish was not significant (Fig. 4). The high variability in the expression of this gene, particularly in WT fish, is a likely explanation for this finding, and suggests that it may also be regulated by other factors in addition to androgen signalling.

As with other zebrafish lines carrying mutations resulting in impaired androgen signalling, cyp11c1-/mutant zebrafish exhibit primarily female pigmentation patterns (Crowder et al. 2018, Zhai et al. 2018, Oakes et al. 2019). Feminisation of anal fin pigmentation appears to be to more pronounced in our study compared to Zhang et al. (2020). This variability may arise from differences in time of analysis and age of fish. In addition to analysis of the anal fin, we have also described feminisation of dorsal fin pigmentation in Cyp11c1-deficient males, this was not formally assessed in the study of Zhang et al., but appeared to be the case in the representative fish presented in their paper. Overall, these findings suggest that androgens may induce the expression of genes important for fin colour patterning during development.

Cyp11c1-deficient adult male zebrafish were infertile in normal breeding scenarios (Table 1); nevertheless, we observed that their sperm could fertilise eggs collected from WT female zebrafish by IVF (Table 2). These findings confirm the results of Zhang et al. (2020), and indicate that Cyp11c1-deficient zebrafish are able to produce mature sperm but are infertile due to another factor, such as impaired breeding behaviour or spermatogenesis, or morphological disruption of the testes or male reproductive tract resulting in impaired sperm release.

Breeding behaviours are decreased in both androgen-deficient and androgen-resistant zebrafish 
A $11 b p$ del-nanos2
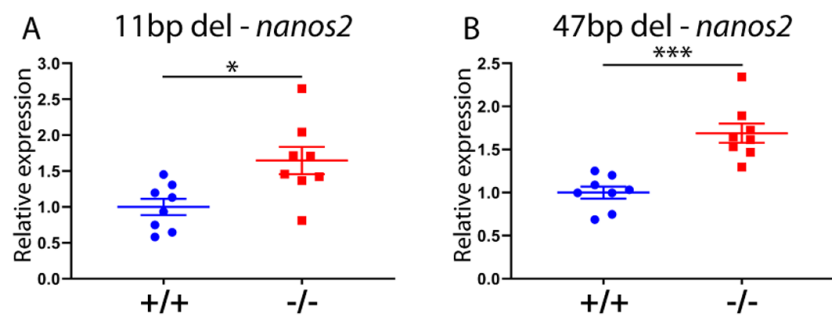

C $\quad 11 \mathrm{bp}$ del-piwil1
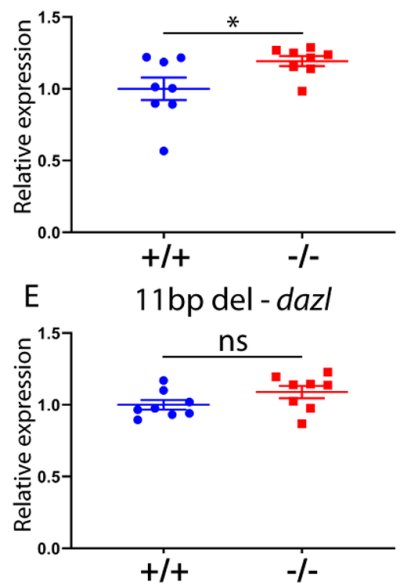

G $\quad 11 b p$ del - sycp3

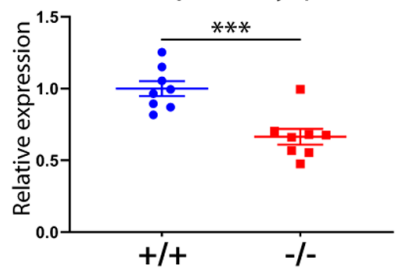

D $\quad 47 \mathrm{bp}$ del - piwil1

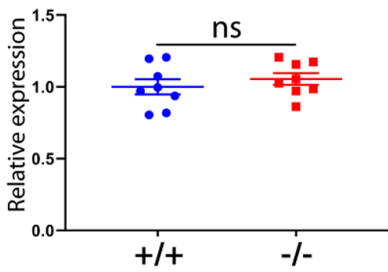

F
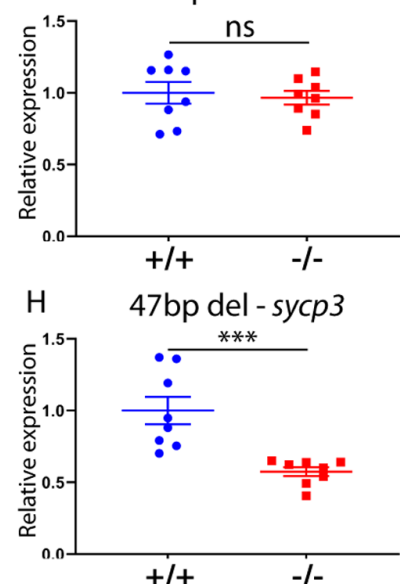

$11 \mathrm{bp}$ del - odf $3 b$
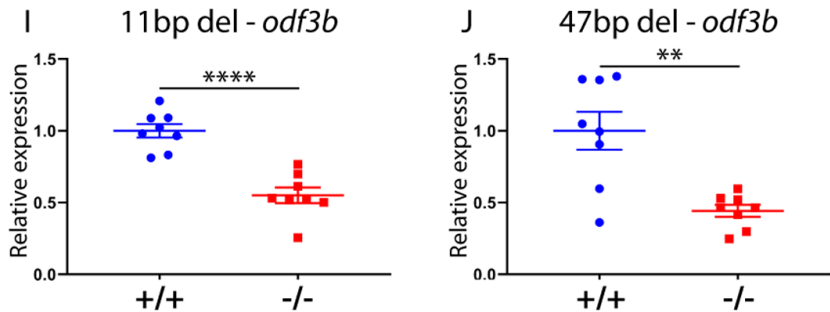

Figure 10

Expression of spermatogenic marker genes in the testes of cyp11c1-1zebrafish. qPCR was employed to measure the expression of marker genes for several spermatogenic stages. The expression of nanos2, a marker of type A undifferentiated spermatogonia, was increased in cyp11c1-/- zebrafish (11 bp $n=8, P=0.0112 ; 47 \mathrm{bp} n=8, P=0.0001)$. The expression of piwil1, a marker of both undifferentiated and differentiated type A spermatogonia was significantly increased in zebrafish homozygous for the $11 \mathrm{bp}$ deletion allele $(n=8, P=0.0405)$, but was not changed in those carrying the $47 \mathrm{bp}$ deletion allele $(n=8, P=0.4385)$. Expression of the type $B$ spermatogonia marker dazl was not affected by mutation of cyp11c1 (11 bp $n=8, P=0.1213$; $47 \mathrm{bp} n=8, P=0.7009$ ). Expression of the spermatocyte and spermatid markers sycp 3 and odf $3 b$ was significantly reduced in the testes of cyp11c1-/- zebrafish. (sycp3: $11 \mathrm{bp}$ $n=8, P=0.0006 ; 47$ bp $n=8, P=0.0008$; odf3b: 11 bp $n=8, P<0.0001$; 47 bp $n=8, P=0.0013)$. Data analysed by unpaired $t$-tests, $* P<0.05$, $\star \star P<0.01, * * \star P<0.001, * * \star * P<0.0001$. A full colour version of this figure is available at https://doi.org/10.1530/JOE-20-0160.
(Yong et al. 2017, Oakes et al. 2019). In this regard, the phenotype of our new cyp11c1-l- mutant zebrafish lines closely resembles that of $f d \times 1 b^{-/-}$mutant zebrafish (Fig. 5) (Oakes et al. 2019). This finding also confirms similar results obtained by Zhang et al. (2020), although different behavioural assays were used. A key difference is our experimental design, in which behavioural trials were repeated several times with the same fish to control for novelty and habituation. Importantly, our results were similar irrespective of trial number, indicating that the phenotype remains the same despite habituation to the technique (Fig. 5).

In addition to analysis of breeding behaviour, we have demonstrated altered locomotor behaviour in Cyp11c1deficient adult zebrafish. To our knowledge, this is the first time adult androgen- and glucocorticoid-deficient zebrafish have been revealed to exhibit such a behavioural phenotype. Cyp11c1-deficient male zebrafish exhibited decreased locomotor activity compared to WT siblings (Fig. 6). Decreased locomotor activity may affect readouts of multiple breeding behaviours; for example, slow swimming fish may have less opportunity for intimate contacts with their tank mate and may not be able to participate effectively in chasing behaviour. Locomotor activity and freezing behaviours have been linked to stress and glucocorticoid signalling in zebrafish; glucocorticoid receptor mutants are known to exhibit freezing behaviour and slower average swim velocities (Ziv et al. 2013). Cortisol deficiency may be responsible for the impaired locomotor behaviour seen in Cyp11c1-deficient zebrafish, but may not fully explain the reduction in breeding behaviours, as mutation of the androgen receptor also produces a similar phenotype (Yong et al. 2017). Overall, the behavioural phenotype of Cyp11c1-deficient zebrafish is likely to result from disruption of both glucocorticoidand androgen-regulated processes.

As previously described in androgen-deficient and androgen-resistant zebrafish lines, we show that androgen signalling is dispensable for definitive testicular differentiation (Crowder et al. 2018, Oakes et al. 2019). In the other recently reported $c y p 11 c 1^{-/-}$mutant zebrafish line, histological examination was restricted to the stage when gonadal differentiation is taking place. Zhang et al. (2020) showed that Cyp11c1-deficiency resulted in prolonged juvenile ovary-to-testis transformation, with degenerating oocytes present long after the normal period of testis differentiation. In contrast to Zhang et al., we examined the histological testicular phenotype of adult cyp11c1 $1^{-/-}$mutant male zebrafish. Our histological examination of adult cyp11c1-/- mutants revealed that 
their testes were highly disorganised: seminiferous tubules were poorly defined, and the quantity of spermatozoa was reduced (Fig. 7). The histological appearance of cyp11c1-l- mutant testes was similar to that described in other zebrafish models of disrupted androgen signalling (Crowder et al. 2018, Oakes et al. 2019), thus providing further confirmation that androgens are required for correct organisation and morphological development or maintenance of the testes. Tubular structure formation in the testes appears to occur during the latter stages of, or after, the period of testicular differentiation in zebrafish, as tubules are not clearly visible until well after the gonad is committed to testis development (van der Ven $\&$ Wester 2003). Overall, it appears that the crucial roles for androgens in zebrafish testicular development are temporal control of gonadal differentiation (Zhang et al. 2020), and subsequent formation and maintenance of correct seminiferous tubule organisation in the juvenile and adult testis.

We previously postulated that Sertoli cell dysfunction may be responsible for the testicular phenotype observed in androgen-deficient zebrafish; several Sertoli cell expressed genes, such as sox9a and inha, were downregulated in $f d \times 1 b^{-/-}$mutant zebrafish, which exhibit a similar phenotype to that described in the present study (Oakes et al. 2019). Sox9a may be of importance in testis tubulogenesis, as a role in this process has been proposed for this gene in a related teleost (Nakamoto et al. 2005). Sox $9 a$ expression was unaffected by mutation of $c y p 11 c 1$, whereas inha was significantly downregulated (Fig. 9). The mechanism by which androgens control appropriate testis tubule morphogenesis or maintenance remains elusive, and is an exciting topic for further study.

Zhang et al. (2020) described that, despite their infertility, Cyp11c1-deficent zebrafish could produce morphologically normal spermatozoa. However, when Cyp11c1-deficient males were subjected to manual gamete expression, a reduced volume of semen was produced in comparison to WTs, indicating impaired spermatogenesis or sperm release in the cyp11c1-/mutants (Zhang et al. 2020). In our Cyp11c1-deficient zebrafish, whole-testes sperm counts were significantly lower than in WT siblings, also indicating impaired spermatogenesis (Fig. 7). To further investigate impaired spermatogenesis we performed novel and in-depth characterisation of spermatogenic defects in Cyp11c1deficient zebrafish by measuring the expression of spermatogenic factors and spermatogenic stage-specific marker genes. We observed significant down-regulation of igf3 and insl3 in Cyp11c1-deficient zebrafish (Fig. 9); both genes are important for the differentiation and proliferation of type A spermatogonia (Nobrega et al. 2015, Assis et al. 2016, Morais et al. 2017). This was reflected by increased expression of nanos2, a marker for type A spermatogonia (Beer \& Draper 2013, Safian et al. 2016), and decreased expression of $s y c p 3$ and $o d f 3 b$, markers of later stages in spermatogenesis (Fig. 10) (Yano et al. 2008, Ozaki et al. 2011, Nobrega et al. 2015). Histological examination also suggested a qualitative increase in the proportion of developing sperm to mature sperm in the testes (Fig. 7). Taken together, these findings support the proposition that, whilst production of type A spermatogonia is normal in cyp11c1-l- mutant testes, the subsequent differentiation of type A spermatogonia is highly androgen-dependent.

In addition to impaired spermatogonial differentiation, entry of type B spermatogonia into meiosis may also be disrupted in cyp11c1-/- mutant zebrafish. Whilst expression of the type B spermatogonia marker dazl was unchanged by mutation cyp11c1, expression of the spermatocyte marker sycp3 was downregulated, as was that of the spermatid marker odf $3 b$ (Fig. 10). Spermatogenic arrest or delay during meiosis has previously been reported in androgen receptor mutant zebrafish (Yu et al. 2018). Sycp3 is a component of the synaptonemal complex, which plays important roles during meiotic prophase, including regulation of chromosome recombination (Page \& Hawley 2004, Syrjänen et al. 2014). Thus, reduced expression of sycp3 in $c y p 11 c 1^{-/-}$mutant spermatocytes could contribute to the impairment of meiosis.

Expression of a small number of genes was characterised at the adult stage in the previously published cyp11c1--- mutant zebrafish line (Zhang et al. 2020). Both the previous mutant and the mutant described herein exhibited decreased expression of the spermatogenic factor insl3. In contrast to observations described by Zhang et al., no change of $d m r t 1$ expression was observed in our cyp11c1-1- mutants (Fig. 9), which is consistent with our previously reported findings in androgen- and cortisol-deficient $f d \times 1 b^{-/-}$mutants (Oakes et al. 2019).

Attempts to manually collect semen from Cyp11c1deficient zebrafish revealed that sperm release may be impaired. However, this technique may not accurately replicate natural ejaculation, and therefore, successful sperm release in natural breeding conditions cannot be ruled out. As sperm release appeared to be impaired, we investigated the structure of the spermatic duct and found that it to be severely hypoplastic in https://joe.bioscientifica.com

https://doi.org/10.1530/JOE-20-0160 (c) 2020 Society for Endocrinology Published by Bioscientifica Ltd.
Printed in Great Britain 
Cyp11c1-deficient zebrafish. Little is known about the development of this structure; however, we have shown here for the first time that its development is highly steroid dependent, and this is likely to be mediated by 11-ketotestosterone. This structure may be comparable to Wolffian duct structures in mammals, however these structures are of different embryological origins and may be analogous in function alone (Shaw \& Renfree 2014, Matthews et al. 2018). Nevertheless, both structures appear to be highly dependent on androgens for their development, as abnormal Wolffian duct structures are frequently seen in complete androgen insensitivity syndrome (Hannema et al. 2006, Barbaro et al. 2007). Wolffian duct structures are also absent in AR knock-out mice (Yeh et al. 2002).

Herein, we have described novel zebrafish lines carrying mutation of cyp11c1, which is crucial for 11-ketotestosterone and cortisol biosynthesis. In addition to confirming several results described in a recently published cyp11c1 zebrafish mutant (Zhang et al. 2020), our study describes novel phenotypes, including testicular disorganisation, hypoplastic spermatic ducts, and impaired locomotor function, as well as characterising spermatogenic defects through the measurement of marker gene expression. As such, our work represents a significant and novel contribution to the literature regarding the roles of steroids in regulation of zebrafish reproduction. cyp11c1-/- mutant zebrafish exhibit a phenotype characteristic of androgen deficiency and represent a novel tool for the investigation of the roles of androgens in male reproductive development and function. The discovery that androgens are essential for spermatic duct morphogenesis in zebrafish is a particularly exciting finding, and will pave the way for further research into this poorly characterised structure.

\section{Supplementary materials}

This is linked to the online version of the paper at https://doi.org/10.1530/ JOE-20-0160.

\section{Declaration of interest}

The authors declare that there is no conflict of interest that could be perceived as prejudicing the impartiality of the research reported.

\section{Funding}

This work was supported by the International Fund Congenital Adrenal Hyperplasia 2017 research grant (to N P K and V T C) and the Deutsche Forschungsgemeinschaft (KR 3363/3-1).

\section{Author contribution statement}

V T C and N P K contributed equally to this work.

\section{References}

Andersen L, Holbech H, Gessbo A, Norrgren L \& Petersen GI 2003 Effects of exposure to 17alpha-ethinylestradiol during early development on sexual differentiation and induction of vitellogenin in zebrafish (Danio rerio). Comparative Biochemistry and Physiology: Toxicology and Pharmacology 134 365-374. (https://doi.org/10.1016/s15320456(03)00006-1)

Assis LHC, Crespo D, Morais RDVS, França LR, Bogerd J \& Schulz RW 2016 INSL3 stimulates spermatogonial differentiation in testis of adult zebrafish (Danio rerio). Cell and Tissue Research 363 579-588. (https://doi.org/10.1007/s00441-015-2213-9)

Baker ME, Nelson DR \& Studer RA 2015 Origin of the response to adrenal and sex steroids: roles of promiscuity and co-evolution of enzymes and steroid receptors. Journal of Steroid Biochemistry and Molecular Biology 151 12-24. (https://doi.org/10.1016/j.jsbmb.2014.10.020)

Barbaro M, Oscarson M, Almskog I, Hamberg H \& Wedell A 2007 Complete androgen insensitivity without Wolffian duct development: the AR-A form of the androgen receptor is not sufficient for male genital development. Clinical Endocrinology 66 822-826. (https://doi. org/10.1111/j.1365-2265.2007.02819.x)

Beer RL \& Draper BW 2013 nanos3 maintains germline stem cells and expression of the conserved germline stem cell gene nanos 2 in the zebrafish ovary. Developmental Biology 374 308-318. (https://doi. org/10.1016/j.ydbio.2012.12.003)

Brion F, Tyler CR, Palazzi X, Laillet B, Porcher JM, Garric J \& Flammarion P 2004 Impacts of 17beta-estradiol, including environmentally relevant concentrations, on reproduction after exposure during embryo-larval-, juvenile- and adult-life stages in zebrafish (Danio rerio). Aquatic Toxicology 68 193-217. (https://doi org/10.1016/j.aquatox.2004.01.022)

Cai K, Hua G, Ahmad S, Liang A, Han L, Wu C, Yang F \& Yang L 2011 Action mechanism of inhibin $\alpha$-subunit on the development of sertoli cells and first wave of spermatogenesis in mice. PLoS ONE 6 e25585. (https://doi.org/10.1371/journal.pone.0025585)

Caulier M, Brion F, Chadili E, Turies C, Piccini B, Porcher JM, Guiguen Y $\&$ Hinfray N 2015 Localization of steroidogenic enzymes and Foxl2a in the gonads of mature zebrafish (Danio rerio). Comparative Biochemistry and Physiology: Part A, Molecular and Integrative Physiology 188 96-106. (https://doi.org/10.1016/j.cbpa.2015.06.016)

Chen SX, Bogerd J, Schoonen NE, Martijn J, De Waal PP \& Schulz RW 2013 A progestin (17alpha,20beta-dihydroxy-4-pregnen-3-one) stimulates early stages of spermatogenesis in zebrafish. General and Comparative Endocrinology 185 1-9. (https://doi.org/10.1016/j. ygcen.2013.01.005)

Crowder CM, Lassiter CS \& Gorelick DA 2018 Nuclear androgen receptor regulates testes organization and oocyte maturation in zebrafish. Endocrinology 159 980-993. (https://doi.org/10.1210/en.2017-00617)

De Waal PP, Wang DS, Nijenhuis WA, Schulz RW \& Bogerd J 2008 Functional characterization and expression analysis of the androgen receptor in zebrafish (Danio rerio) testis. Reproduction 136 225-234. (https://doi.org/10.1530/REP-08-0055)

Eachus H, Zaucker A, Oakes JA, Griffin A, Weger M, Guran T, Taylor A, Harris A, Greenfield A, Quanson JL et al. 2017 Genetic disruption of 21-hydroxylase in zebrafish causes interrenal hyperplasia. Endocrinology 158 4165-4173. (https://doi.org/10.1210/en.2017-00549)

Fetter E, Smetanova S, Baldauf L, Lidzba A, Altenburger R, Schuttler A \& Scholz S 2015 Identification and characterization of androgenresponsive genes in zebrafish embryos. Environmental Science and Technology 49 11789-11798. (https://doi.org/10.1021/acs. est.5b01034)

Gregory SJ \& Kaiser UB 2004 Regulation of gonadotropins by inhibin and activin. Seminars in Reproductive Medicine 22 253-267. (https://doi. org/10.1055/s-2004-831901)

Griffin A, Parajes S, Weger M, Zaucker A, Taylor AE, O’Neil DM, Muller F \& Krone N 2016 Ferredoxin 1b (Fdx1b) is the essential mitochondrial 
redox partner for cortisol biosynthesis in zebrafish. Endocrinology 157 1122-1134. (https://doi.org/10.1210/en.2015-1480)

Hannema SE, Scott IS, Rajpert-De Meyts E, Skakkebaek NE, Coleman N \& Hughes IA 2006 Testicular development in the complete androgen insensitivity syndrome. Journal of Pathology 208 518-527. (https://doi. org/10.1002/path.1890)

Larsen MG \& Baatrup E 2010 Functional behavior and reproduction in androgenic sex reversed zebrafish (Danio rerio). Environmental Toxicology and Chemistry 29 1828-1833. (https://doi.org/10.1002/etc.214)

Lau ES, Zhang Z, Qin M \& Ge W 2016 Knockout of zebrafish ovarian aromatase gene (cyp19a1a) by TALEN and CRISPR/Cas9 leads to all-male offspring due to failed ovarian differentiation. Scientific Reports 6 37357. (https://doi.org/10.1038/srep37357)

Lee SLJ, Horsfield JA, Black MA, Rutherford K, Fisher A \& Gemmell NJ 2017 Histological and transcriptomic effects of 17alphamethyltestosterone on zebrafish gonad development. BMC Genomics 18 557. (https://doi.org/10.1186/s12864-017-3915-z)

Li N, Oakes JA, Storbeck KH, Cunliffe VT \& Krone NP 2020 The P450 side chain cleavage enzyme Cyp11a2 facilitates steroidogenesis in zebrafish. Journal of Endocrinology 244 309-321. (https://doi. org/10.1530/JOE-19-0384)

Liew WC, Bartfai R, Lim Z, Sreenivasan R, Siegfried KR \& Orban L 2012 Polygenic sex determination system in zebrafish. PLoS ONE 7 e34397. (https://doi.org/10.1371/journal.pone.0034397)

Livak KJ \& Schmittgen TD 2001 Analysis of relative gene expression data using real-time quantitative PCR and the 2(-Delta Delta C(T)) method. Methods 25 402-408. (https://doi.org/10.1006/meth.2001.1262)

Matthews JL, Murphy JM, Carmichael C, Yang H, Tiersch T, Westerfield M \& Varga ZM 2018 Changes to extender, cryoprotective medium, and in vitro fertilization improve zebrafish sperm cryopreservation. Zebrafish 15 279-290. (https://doi.org/10.1089/ zeb.2017.1521)

Menke AL, Spitsbergen JM, Wolterbeek AP \& Woutersen RA 2011 Normal anatomy and histology of the adult zebrafish. Toxicologic Pathology 39 759-775. (https://doi.org/10.1177/0192623311409597)

Miller WL \& Auchus RJ 2011 The molecular biology, biochemistry, and physiology of human steroidogenesis and its disorders. Endocrine Reviews 32 81-151. (https://doi.org/10.1210/er.2010-0013)

Morais RDVS, Crespo D, Nobrega RH, Lemos MS, Van De Kant HJG De Franca LR, Male R, Bogerd J \& Schulz RW 2017 Antagonistic regulation of spermatogonial differentiation in zebrafish (Danio rerio) by Igf3 and Amh. Molecular and Cellular Endocrinology 454 112-124. (https://doi.org/10.1016/j.mce.2017.06.017)

Morthorst JE, Holbech H \& Bjerregaard P 2010 Trenbolone causes irreversible masculinization of zebrafish at environmentally relevant concentrations. Aquatic Toxicology 98 336-343. (https://doi. org/10.1016/j.aquatox.2010.03.008)

Nakamoto M, Suzuki A, Matsuda M, Nagahama Y \& Shibata N 2005 Testicular type Sox9 is not involved in sex determination but might be in the development of testicular structures in the medaka, Oryzias latipes. Biochemical and Biophysical Research Communications $\mathbf{3 3 3}$ 729-736. (https://doi.org/10.1016/j.bbrc.2005.05.158)

Nobrega RH, Morais RD, Crespo D, De Waal PP, De Franca LR, Schulz RW \& Bogerd J 2015 Fsh stimulates spermatogonial proliferation and differentiation in zebrafish via Igf3. Endocrinology 156 3804-3817. (https://doi.org/10.1210/en.2015-1157)

Oakes JA, Li N, Wistow BRC, Griffin A, Barnard L, Storbeck KH, Cunliffe VT $\&$ Krone NP 2019 Ferredoxin 1b deficiency leads to testis disorganization, impaired spermatogenesis and feminization in zebrafish. Endocrinology 160 2401-2416. (https://doi.org/10.1210/en.2019-00068)

O'Reilly MW, Kempegowda P, Jenkinson C, Taylor AE, Quanson JL, Storbeck KH \& Arlt W 2017 11-Oxygenated C19 steroids are the predominant androgens in polycystic ovary syndrome. Journal of Clinical Endocrinology and Metabolism 102 840-848. (https://doi. org/10.1210/jc.2016-3285)
Orn S, Holbech H \& Norrgren L 2016 Sexual disruption in zebrafish (Danio rerio) exposed to mixtures of 17alpha-ethinylestradiol and 17beta-trenbolone. Environmental Toxicology and Pharmacology 41 225-231. (https://doi.org/10.1016/j.etap.2015.12.010)

Ozaki Y, Saito K, Shinya M, Kawasaki T \& Sakai N 2011 Evaluation of Sycp3, Plzf and cyclin B3 expression and suitability as spermatogonia and spermatocyte markers in zebrafish. Gene Expression Patterns 11 309-315. (https://doi.org/10.1016/j.gep.2011.03.002)

Page SL \& Hawley RS 2004 The genetics and molecular biology of the synaptonemal complex. Annual Review of Cell and Developmental Biology 20 525-558. (https://doi.org/10.1146/annurev. cellbio.19.111301.155141)

Poon SK, So WK, Yu X, Liu L \& Ge W 2009 Characterization of inhibin alpha subunit (Inha) in the zebrafish: evidence for a potential feedback loop between the pituitary and ovary. Reproduction $\mathbf{1 3 8}$ 709-719. (https://doi.org/10.1530/REP-09-0198)

Safian D, Morais RD, Bogerd J \& Schulz RW 2016 Igf binding proteins protect undifferentiated spermatogonia in the zebrafish testis against excessive differentiation. Endocrinology 157 4423-4433. (https://doi. org/10.1210/en.2016-1315)

Schiffer L, Anderko S, Hannemann F, Eiden-Plach A \& Bernhardt R 2015 The CYP11B subfamily. Journal of Steroid Biochemistry and Molecular Biology 151 38-51. (https://doi.org/10.1016/j.jsbmb.2014.10.011)

Shaw G \& Renfree MB 2014 Wolffian duct development. Sexual Development 8 273-280. (https://doi.org/10.1159/000363432)

Siegenthaler PF, Zhao Y, Zhang K \& Fent K 2017 Reproductive and transcriptional effects of the antiandrogenic progestin chlormadinone acetate in zebrafish (Danio rerio). Environmental Pollution 223 346-356. (https://doi.org/10.1016/j.envpol.2017.01.031)

Sun D, Zhang Y, Wang C, Hua X, Zhang XA \& Yan J 2013 Sox9-related signaling controls zebrafish juvenile ovary-testis transformation. Cell Death and Disease 4 e930. (https://doi.org/10.1038/cddis.2013.456)

Syrjänen JL, Pellegrini L \& Davies OR 2014 A molecular model for the role of SYCP3 in meiotic chromosome organisation. eLife 3 e02963. (https://doi.org/10.7554/eLife.02963)

Tang H, Chen Y, Wang L, Yin Y, Li G, Guo Y, Liu Y, Lin H, Cheng CHK \& Liu X 2018 Fertility impairment with defective spermatogenesis and steroidogenesis in male zebrafish lacking androgen receptor. Biology of Reproduction 98 227-238. (https://doi.org/10.1093/biolre/iox165)

Tokarz J, Moller G, Hrabe De Angelis M \& Adamski J 2015 Steroids in teleost fishes: a functional point of view. Steroids 103 123-144. (https://doi.org/10.1016/j.steroids.2015.06.011)

Uchida D, Yamashita M, Kitano T \& Iguchi T 2002 Oocyte apoptosis during the transition from ovary-like tissue to testes during sex differentiation of juvenile zebrafish. Journal of Experimental Biology 205 711-718.

Van Der Ven L \& Wester P 2003 Histology and Histopathology Atlas of the Zebrafish. Bilthoven, Netherlands: National Institute of Public Health and the Environment of the Netherlands (RIVM). (available at: https://zfin.org/hh_atlas/)

Wang XG, Bartfai R, Sleptsova-Freidrich I \& Orban L 2007 The timing and extent of 'juvenile ovary' phase are highly variable during zebrafish testis differentiation. Journal of Fish Biology 70 33-44. (https://doi. org/10.1111/j.1095-8649.2007.01363.x)

Webster KA, Schach U, Ordaz A, Steinfeld JS, Draper BW \& Siegfried KR 2017 Dmrt1 is necessary for male sexual development in zebrafish. Developmental Biology 422 33-46. (https://doi.org/10.1016/j. ydbio.2016.12.008)

Westerfield M 2000 The Zebrafish Book: A Guide for the Laboratory Use of Zebrafish (Danio rerio), 4th ed. Eugene, OR, USA: University of Oregon Press.

Yano A, Suzuki K \& Yoshizaki G 2008 Flow-cytometric isolation of testicular germ cells from rainbow trout (Oncorhynchus mykiss) carrying the green fluorescent protein gene driven by trout vasa regulatory regions. Biology of Reproduction $\mathbf{7 8}$ 151-158. (https://doi. org/10.1095/biolreprod.107.064667) https://joe.bioscientifica.com https://doi.org/10.1530/JOE-20-0160 (c) 2020 Society for Endocrinology Published by Bioscientifica Ltd. Printed in Great Britain 
Yeh S, Tsai M-Y, Xu Q, Mu X-M, Lardy H, Huang K-E, Lin H, Yeh S-D, Altuwaijri S, Zhou X et al. 2002 Generation and characterization of androgen receptor knockout (ARKO) mice: an in vivo model for the study of androgen functions in selective tissues. PNAS 99 1349813503. (https://doi.org/10.1073/pnas.212474399)

Yin Y, Tang H, Liu Y, Chen Y, Li G, Liu X \& Lin H 2017 Targeted disruption of aromatase reveals dual functions of cyp19a1a during sex differentiation in zebrafish. Endocrinology 158 3030-3041. (https:// doi.org/10.1210/en.2016-1865)

Yong L, Thet Z \& Zhu Y 2017 Genetic editing of the androgen receptor contributes to impaired male courtship behavior in zebrafish. Journal of Experimental Biology 220 3017-3021. (https://doi.org/10.1242/ jeb.161596)

Yu G, Zhang D, Liu W, Wang J, Liu X, Zhou C, Gui J \& Xiao W 2018 Zebrafish androgen receptor is required for spermatogenesis and maintenance of ovarian function. Oncotarget 9 24320-24334. (https:// doi.org/10.18632/oncotarget.24407)

Zhai G, Shu T, Xia Y, Lu Y, Shang G, Jin X, He J, Nie P \& Yin Z 2018 Characterization of sexual trait development in cyp17a1-deficient zebrafish. Endocrinology 159 3549-3562. (https://doi.org/10.1210/ en.2018-00551)

Zhang Q, Ye D, Wang H, Wang Y, Hu W \& Sun Y 2020 Zebrafish cyp11c1 knockout reveals the roles of 11-ketotestosterone and cortisol in sexual development and reproduction. Endocrinology 161 bqaa048. (https://doi.org/10.1210/endocr/bqaa048)

Ziv L, Muto A, Schoonheim PJ, Meijsing SH, Strasser D, Ingraham HA, Schaaf MJ, Yamamoto KR \& Baier H 2013 An affective disorder in zebrafish with mutation of the glucocorticoid receptor. Molecular Psychiatry 18 681-691. (https://doi.org/10.1038/ mp.2012.64)

Received in final form 10 July 2020

Accepted 14 August 2020

Accepted Manuscript published online 17 August 2020 (c) 2020 Society for Endocrinology Published by Bioscientifica Ltd. 